\title{
Agent-based model of land system: Theory, application and modelling framework
}

\author{
DAI Erfu ${ }^{1,3}$, MA Liang ${ }^{1,3}$, YANG Weishi, ${ }^{2,4}$, WANG Yahui ${ }^{1,3}$, YIN Le $e^{2,3}$, \\ TONG Miao ${ }^{1,3}$
}

1. Lhasa Plateau Ecosystem Research Station, Key Laboratory of Ecosystem Network Observation and Modeling, Institute of Geographic Sciences and Natural Resources Research, CAS, Beijing 100101, China;

2. Key Laboratory of Land Surface Pattern and Simulation, Institute of Geographic Sciences and Natural Resources Research, CAS, Beijing 100101, China;

3. University of Chinese Academy of Sciences, Beijing 100049, China;

4. School of Geography and Planning, Sun Yat-Sen University, Guangzhou 510275, China

\begin{abstract}
Land change science has become an interdisciplinary research direction for understanding human-natural coupling systems. As a process-oriented modelling approach, agent based model (ABM) plays an important role in revealing the driving forces of land change and understanding the process of land change. This paper starts from three aspects: The theory, application and modeling framework of ABM. First, we summarize the theoretical basis of $A B M$ and introduce some related concepts. Then we expound the application and development of $A B M$ in both urban land systems and agricultural land systems, and further introduce the case study of a model on Grain for Green Program in Hengduan Mountainous region, China. On the basis of combing the ABM modeling protocol, we propose the land system $A B M$ modeling framework and process from the perspective of agents. In terms of urban land use, ABM research initially focused on the study of urban expansion based on landscape, then expanded to issues like urban residential separation, planning and zoning, ecological functions, etc. In terms of agricultural land use, ABM application presents more diverse and individualized features. Research topics include farmers' behavior, farmers' decision-making, planting systems, agricultural policy, etc. Compared to traditional models, ABM is more complex and difficult to generalize beyond specific context since it relies on local knowledge and data. However, due to its unique bottom-up model structure, ABM has an indispensable role in exploring the driving forces of land change and also the impact of human behavior on the environment.
\end{abstract}

Keywords: land system; land use; land change science; land change model; agent-based model; modeling framework

Received: 2020-03-31 Accepted: 2020-06-30

Foundation: National Natural Science Foundation of China, No.41571098, No.41530749; National Key R\&D Program of China, No.2017YFC1502903, No.2018YFC1508805

Author: Dai Erfu (1972-), PhD and Professor, specialized in comprehensive study of physical geography, simulation of LUCC, and climate change. E-mail: daief@igsnrr.ac.cn 


\section{Introduction}

Land change has great impact on regional environment and human well-being, and is also an important driver of global change (Lambin and Geist, 2006; Verburg et al., 2015). Land system provides food, energy, resources and other goods and services for survival and development of human society. A deep understanding of land system dynamics can promote land and space management and regional planning. Recently, with the emergence and development of land change science, researchers from multi-discipline carried out a number of works (Turner et al., 2007; Rounsevell et al., 2012; Verburg et al., 2013; Müller and Munroe, 2014). Model and simulation is an important tool for studying changes and dynamics of land use and land cover change (Verburg et al., 2004; Brown et al., 2013; Li et al., 2017; Verburg et al., 2019). The role of land change model includes analyzing the relation between observed patterns and the process that produced them, as well as simulating and predicting future land change under scenarios of different policies. The results of land change model can be used as input of ecosystem models.

The real world is a complex system composed of a large number of decision-making agents. One of the most important challenges facing contemporary sciences is understanding complex systems (Grimm et al., 2005). As the interface between human society and physical environment, land system is a typical complex system (Yu et al., 2011; Verburg et al., 2013; Dai and Ma, 2018). With the application of complexity science and methods, researchers in the field of land change models are committed to explicitly characterizing human decision-making behaviors by coupling natural and human systems (Brown et al., 2013). Agent-based model (ABM) is specially used to simulate the decision-making behaviors of agents at micro level, and to achieve emergence of macro patterns by representing a large number of agents interactions and feedbacks between agents and environment. This method adopts a bottom-up framework, focusing on representing of the behavior rules, avoiding the understanding and analysis of the entire system, further avoiding the difficulty of quantitative description of complex system by mathematical or physical models. As a result, ABM is an effective way to study complex systems and has become one of the development directions of land change modeling approaches (Parker et al., 2003; Macal and North, 2010; An, 2012; Le Page et al., 2013).

This paper starts from ABM theory, application, and modeling framework of land system. Based on summary and review, we conduct a case study and propose a modeling framework. The content includes four parts: 1) ABM theory, we introduce ABM theory and related concepts. 2) ABM applications, we review ABM application in urban and agricultural land systems, followed by a case study of Chinese Grain for Green Program ABM. 3) Modeling framework, based on existing model communication methods like ODD protocols, we propose modeling framework from the agent perspective of land system. 4) Discussion, challenges and future research directions are discussed.

\section{ABM theoretical basis and related concepts}

\subsection{Complexity theory and $\mathrm{ABM}$}

Originated from general systems theory, complexity theory has multi-disciplinary back- 
ground. It absorbs theories from mathematics, physics, genetic biology and social sciences during the process of development. Composed by many components that interact with each other, complex systems have features include path-dependence, criticality, spatial self-organization and emergence (An, 2012). One of the hallmarks of complexity is the emergence of macro patterns from local interactions (Green and Sadedin, 2005). Complex interactions may lead to chaos, making the system prediction difficult. In modeling complex systems, top-down approaches often have difficulty capturing local interactions. With the development of computer simulation technology, a bottom-up approach can better characterize complex systems.

The agent-based model is a modeling method based on complexity theory. Farmer et al. defined ABM as a computer simulation of a large number of decision-making agents and institutions interacting through prescribing rules (Farmer and Foley, 2009). Agents in ABM are autonomous individuals endowed with attributes and behaviors in computer simulations. They can execute decisions, can learn and adapt based on their memories. ABM allows the modeler to explore the spatial emergence patterns produced by interactions between agents, between agents and environment, and between the environments.

\subsection{Related concepts}

\section{(1) Cellular automata}

As a bottom-up modeling approach, ABM traces back to cellular automata (CA) (Wolfram, 2002; Zhou et al., 2009). CA represents landscape as grids, where each grid has a fixed location and attributes that characterize the environment, such as vegetation or terrain (Green and Sadedin, 2005). ABM maintains the core concept of CA, that is, the behaviors of individuals or grids determine the system-level results (North and Macal, 2007). Both of them are considered as ideal methods for representing complex systems, because both are network systems composed of many simple components (cell grids or agents) without central control, which simulate complex dynamics through simple rules. Compared with ABM, CA has obvious limitations in representing the decision-making behaviors of the government and residents. CA also has shortcomings when dealing with mobile objects such as pedestrians, household settlements, and company locations (Benenson and Torrens, 2004). Dynamic rules in CA are often fixed interactions, while interactions in ABM can be changed as the model runs, because the latter defines rules at the agent level rather than the spatial grid level (Johnston, 2013; Li et al., 2017). Agents not only have neighbor interactions, but also have the ability to learn and evolve.

(2) Individual based model

ABM is also derived from individual based model (IBM) in population ecology. Ecology has a long tradition of bottom-up simulation (Grimm et al., 2005). IBM was widely used in ecological research around 1990, while ABM was widely used in social sciences about 10 years later (Grimm, 1999; Schulze et al., 2017). The name of IBM was maintained in subsequent ecological research. IBM programmatically represents each plant or animal as an individual capable of responding in a certain way and is used to simulate moving animals or people interacting with the landscape (Green and Sadedin, 2005). Similar to ABM, IBM understands system features at the population or community level from individuals and local interactions. Therefore, using the concepts and methods established in IBM in ecology can 
better develop ABM in social science such as land system research.

(3) Object-oriented programming

Essentially ABM is a computer model. Models in natural sciences are usually quantitative, but computer languages allow the description of qualitative conditions (such as if... then...), thereby allowing the expression of behavior rules in ABM. Scientists believe that ABM is related to object-oriented programming (OOP) in computer sciences in the 1980s (An, 2012). In OOP, each object can receive data, process data and pass data to other objects. Objects with consistent data structures and behaviors are abstracted into class. Therefore, OOP logic matches ABM logic in many ways. In fact, ABMs are often built with OOP languages such as $\mathrm{C}++$ and Java (An et al., 2005). With the development of OOP in the field of programming, some scholars believe that almost all computer simulations in the future will be ABM (North and Macal, 2007).

\section{Application of $\mathrm{ABM}$ in land system and case study}

$\mathrm{ABM}$ has a very wide range of applications, including business organization, economics, infrastructure, group events, society and culture, terrorism, military, biology, and ecosystems (Allan, 2010). In the field of geography and environment, there are many case studies such as pedestrian models (Batty, 2001a; Batty et al., 2003), fishing strategies (Little et al., 2009; Cabral et al., 2010), urban development (Brown and Robinson, 2006; Liu et al., 2006; Filatova et al., 2009a), animal population (Perez and Dragicevic, 2010; Topping et al., 2010), transportation (Bravo et al., 2010; Ascensao et al., 2013), agricultural economy (Castella et al., 2005; Le et al., 2008; Valbuena et al., 2010a; Valbuena et al., 2010b), tourism (Johnson and Sieber, 2011; Mao et al., 2014), epidemic spread (Dion et al., 2011), forest management (Bone and Dragicevic, 2010), water resources management (Schluter and Pahl-Wostl, 2007) and many others. Many of these studies involve land systems directly or indirectly.

\subsection{Urban land use change models}

Cities are the areas where human use the land most intensively on the earth surface. Urban land changes during the development process of the city. Schelling and Sakoda were the pioneers in the research of decision-making of urban land use agent. They independently proposed models in 1970 to simulate the residential segregation caused by individuals' slight difference in preference to neighbors (Benenson and Torrens, 2004). They did not even use a computer at the time; they used a chessboard for simulation. Batty did not explicitly adopt the concept of ABM when simulating landscape changes of multi-core cities, but considered the process of residential location by residents (Batty, 2001b). Brown and Robinson (2006) explicitly built an ABM to simulate the urban expansion of southern Michigan. Resident questionnaires were used to parameterize the model. Liu et al. (2006) built an ABM of three types of agents including residents, real estate companies and the government to simulate the urban expansion of Haizhu District, Guangzhou, and they compared the results with CA. They further expanded their research area to the entire Pearl River Delta region and simulated urban land change under various planning scenarios (Li and Liu, 2007; Li and Liu, 2008).

With the deepening of research, urban ABM has become more meticulous and diversified, and sociological and economic theories have been widely applied. Haase et al. $(2010 ; 2012)$ 
studied the phenomenon of urban shrinkage in Leipzig, eastern Germany. Shin and Fossett (2008) improved the satisfaction evaluation method based on the Schelling model, and further studied the residential segregation of different races in American cities. Parker and Filatova (2008) and Filatova et al. (2009a; 2009b) introduced microeconomics and space economics models into an ABM, and simulated the urban land market. The agents are buyers and sellers of land transactions and the simulation results are consistent with the classic Thünen model. Based on their research, Huang et al. (2013) and Sun et al. (2014) further explored the role of agent heterogeneity in the land market, with consideration of the effects of budget constraints and biding. Magliocca et al. (2011) further considered the real estate market and proposed a model coupled real estate market and land market. Li et al. (2013) simulated the spatial population dynamics of the manufacturing city of Dongguan, Guangdong Province based on labor economics. Wahyudi et al. (2019) directly took real estate developers as agents, and researched the impact of developer types, characteristics and behaviors on Jakarta's urban expansion. In recent years, with the study of the coupled human-nature system, coupling land use models with ecological models has become an important research direction. For example, Robinson et al. (2013) combined the ABM suburban development model DEED with the ecosystem process model BIOME-BGC to explore the impact of suburban land management strategies on ecosystem functions.

Methods in ABM for studying urban development issues are also constantly being developed and improved, and gradually tend to couple ABM with other approaches, such as Bayesian networks, genetic algorithms, analytic hierarchy process, game theory (Kocabas and Dragicevic, 2009; Zhang et al., 2010; Tian and Qiao, 2014; Tan et al., 2015). Most of the time, ABM is built as a framework, and another method is used to determine the agents' decision rules. In addition, typical urban land models use square grids as simulate units, and some modelers have explored vector-based simulation (Dahal and Chow, 2014).

\subsection{Agricultural land use change models}

In addition to cities, agriculture is another significant way human use land. Farmers cut down forests, plant crops, and run farms. Their behaviors are more direct and diversified than urban land use behaviors. In addition, compared with the city, the rural landscape has a more prominent interaction between human and nature. Therefore, researchers often build agricultural ABM based on specific natural conditions, socio-economic conditions, and local policies. They usually investigate local knowledge in the research area. Research goals are generally to address specific issues of the region or to provide support for agricultural policymaking.

Deadman et al. (2004) built a household farm model LUCITA, which simulates the development of family farms in Amazon rainforest and land use behaviors of farmers. Castella et al. (2005) combined ABM and role-playing game (RPG) to build a participatory agent-based model SAMBA and simulate land use change under the institutional change in the mountainous region of northern Vietnam. Schmit and Rounsevell (2006) studied the impact of farmers' imitation behaviors in crop planting on agricultural land use. Le Page et al. (2013) developed a land use dynamic simulator LUDAS, which takes population and landscape as spatial self-organizing agents, and they conducted a comprehensive human-land system simulation with farmer decision-making and forest yield. Brady et al. (2009) com- 
bined $\mathrm{ABM}$ and landscape index to study the impact of European agricultural policy and farm reform on landscape. Valbuena et al. (2010a; 2010b) studied the impact of farmers' decision-making on land change and landscape structures in rural areas in Australia and the Netherlands, and made predictions based on scenarios. They also extended their local scale model to regional scales and explored the trajectory of agricultural development (Valbuena et al., 2010c). Murray-Rust et al. (2014) developed an agricultural land use simulation open framework Aporia, which adopts a modular design and can be coupled with multiple vegetation models for evaluating ecosystem service indicators. Bakker et al. (2015) built the rural land exchange model (RULEX), and simulated land use change caused by land exchange between landowners in the eastern Netherlands. Villamor and Noordwijk (2016) combined $\mathrm{ABM}$ and RPG to study the impact of gender on land use decisions in rubber agriculture in Sumatra. They concluded that men tend to maintain a mixed agricultural and forestry economy, while women are more inclined to transform land into the more profitable one.

In addition, some researchers studied the effectiveness of ecologic protection policies, such as China's natural forest protection project (Chen et al., 2012; Chen et al., 2014) and the Grain for Green Program (Sun and Muller, 2013; Yang, 2019). Chen et al. (2014) studied the effect of the subsidy policy of natural forest protection project on reducing harvesting behaviors and restoring forestland in Wolong Nature Reserve, Sichuan Province, China. They also predicted land use under different subsidy scenarios. The types of land use studied in these cases may be forestland or grassland instead of only agricultural land, but the agents are still farmers.

Different agricultural ABMs have obvious local characteristics. They are more like case diagnosis of specific issues in regional sustainable development or natural resource management. In recent years, participatory modeling has gained popularity. The researchers organized different stakeholders such as local villagers, governments, and enterprises to participate directly in the formulation of model rules. They reproduce the decision-making process through interactive methods like games.

\subsection{ABM case study of Grain for Green Program}

The Grain for Green Program (GGP) as a representative of ecological engineering has had an important impact on land use patterns in China. Hengduan mountainous region is a key area to implement the GGP. Based on the ABM modeling framework and process, we built a spatial ABM for the GGP implementation. Taking Tongdu Town, Dongchuan District, Yunnan Province as the research area, based on census data, geographic data, and survey data, we simulated the GGP implementation process from 2010 to 2015. Our simulation results also include the annual income of farmers and households, and the spatial distribution of farmers' and government's willingness to return cropland (Yang, 2019).

We represented three types of agents in the model including farmers, farmer households and the government. Farmers have attributes such as age, fertility, and mortality. Households are basic units of decision-making. Households and the government each determine their willingness to return the cropland in accordance with the changes in expected income and policies. On one hand, by calculating the annual income from working after participating in the GGP and the annual income from planting before participating in the GGP, combined with the subsidy from the government, we can get the income change, and further the will- 
ingness of the households to return their land. On the other hand, the government determines the willingness considering the slope, soil fertility, ecological importance and ease of project implementation. Then we calculate the total willingness based on the willingness of two sides. The parcel is used as simulation space unit. Based on the government's annual quota of the GGP, any cultivated land in the area that meets the requirements of the GGP (slope greater than 25 degrees) would be sorted in descending order to the total willingness and implement the returning until the quota is completed. According to the model framework and decision rules of each agent type, we built a computer model with Java in the Repast (Figure 1). The model was parameterized with survey data. We simulated land use change under the GGP policy from 2011 to 2015 (Figure 2). Using point-to-point accuracy verification of remote sensing data, the accuracy of the model reaches $91.12 \%$.

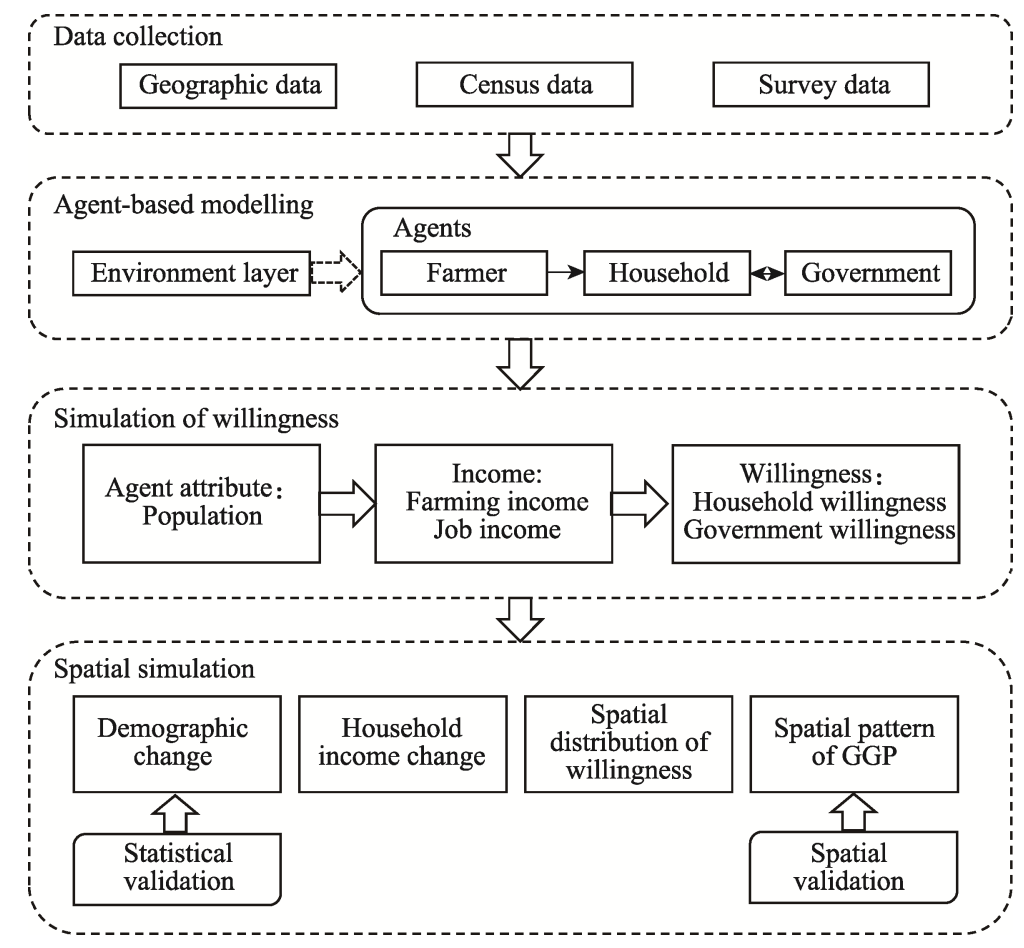

Figure 1 Framework of regional spatial simulation of Grain for Green Program implementation

(a)

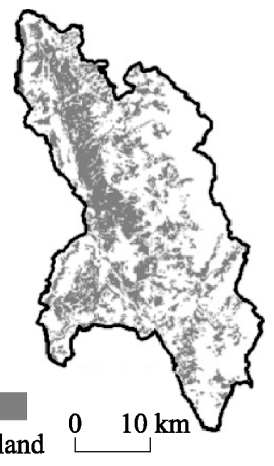

(b)

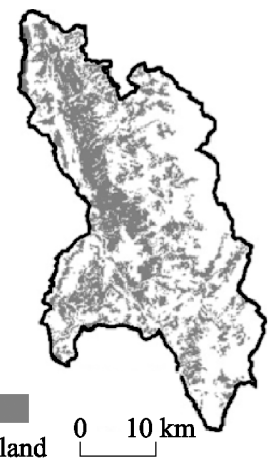

(c)

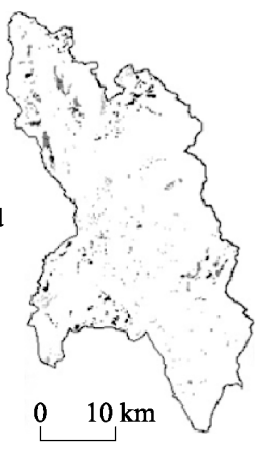

Figure 2 Results of Grain for Green Program in Tongdu Town. Initial spatial pattern of cropland in 2010 (a), simulated spatial pattern of cropland in 2015 (b), and returned farmland from 2011 to 2015 (c) 
Different from the traditional land use models, this model directly represents the agents' behaviors of decision-making and the parameters are defined at agent level. Its advantage lies in reproducing the process of returning cropland to forest instead of the phenomenon. Subsequent research can further simulate the implementation of GGP and land change patterns under different policy scenarios. The results can provide a basis for policy formulation and promote the sustainable development of environment in mountain areas.

\section{ABM modeling framework}

\subsection{Model communication and ODD protocols}

ABM is more of a modeling concept than technology. Multidisciplinary researchers build models to solve specific problems in different regions. They differ very much in theoretical basis, structures and details. The feature of ABM that mixing qualitative and quantitative methods in rule setting means it cannot be expressed in mathematical language transparently. Therefore, effective communication has always been a problem faced by researchers. Using Unified Modeling Language (UML) in software development can promote model communication, but it is not a specialized solution for ABM. Janssen et al. (2008) launched the OpenABM online forum in 2008 for model publishing and code sharing. Model sharing promotes communication, but researchers use different programming languages and platforms. Most ABM researchers are still inefficient when facing the source code directly.

To facilitate model evaluation, comparison and communication, ABM researchers believe that a specific protocol should be followed at the beginning of model building. Grimm et al (2006) proposed a protocol for building IBM/ABM, that is ODD (Overview-Design concepts-Details) protocol, which includes seven elements and 51 guiding questions to help modelers build ABM. Many subsequent studies designed their model according to ODD protocol (Bert et al., 2011; Schreinemachers and Berger, 2011; An et al., 2014). As ODD protocol was based on ecological perspective, Muller et al. (2013) further proposed the $\mathrm{ODD}+\mathrm{D}$ (Decision-making) protocol to better integrate human decision-making into the framework. Recently, as ABM design increasingly used empirical data, Laatabi et al. (2018) proposed the ODD $+2 \mathrm{D}$ (Decision + Data) protocol to refine the data part of the framework (Figure 3). ODD series protocols have been widely used in complex systems and agent-based modeling research groups, which standardize model communication and evaluation. Specifically in the field of land use change model, Parker et al. (2008) proposed the MR POTATOHEAD model design conceptual framework, which divided the model into six parts: information/data, interface with other models, demographics, land use decision making, land exchange and model operation. This framework can be used as a guide for designing models and also as a means of analyzing models. However, its application is not as extensive as ODD series protocols.

\subsection{Agent-based modeling framework and implementation process of land system from the agent perspective}

For modelers, the most important point that ABM is different from other models is to represent reality from the agent perspective. Based on this, we propose the agent-based modeling framework of land system from agent perspective (Figure 4). The modeling process starts 
from the micro agent perspective, theoretical basis and empirical basis, focusing on the constructions of agent subsystem and environment subsystem.

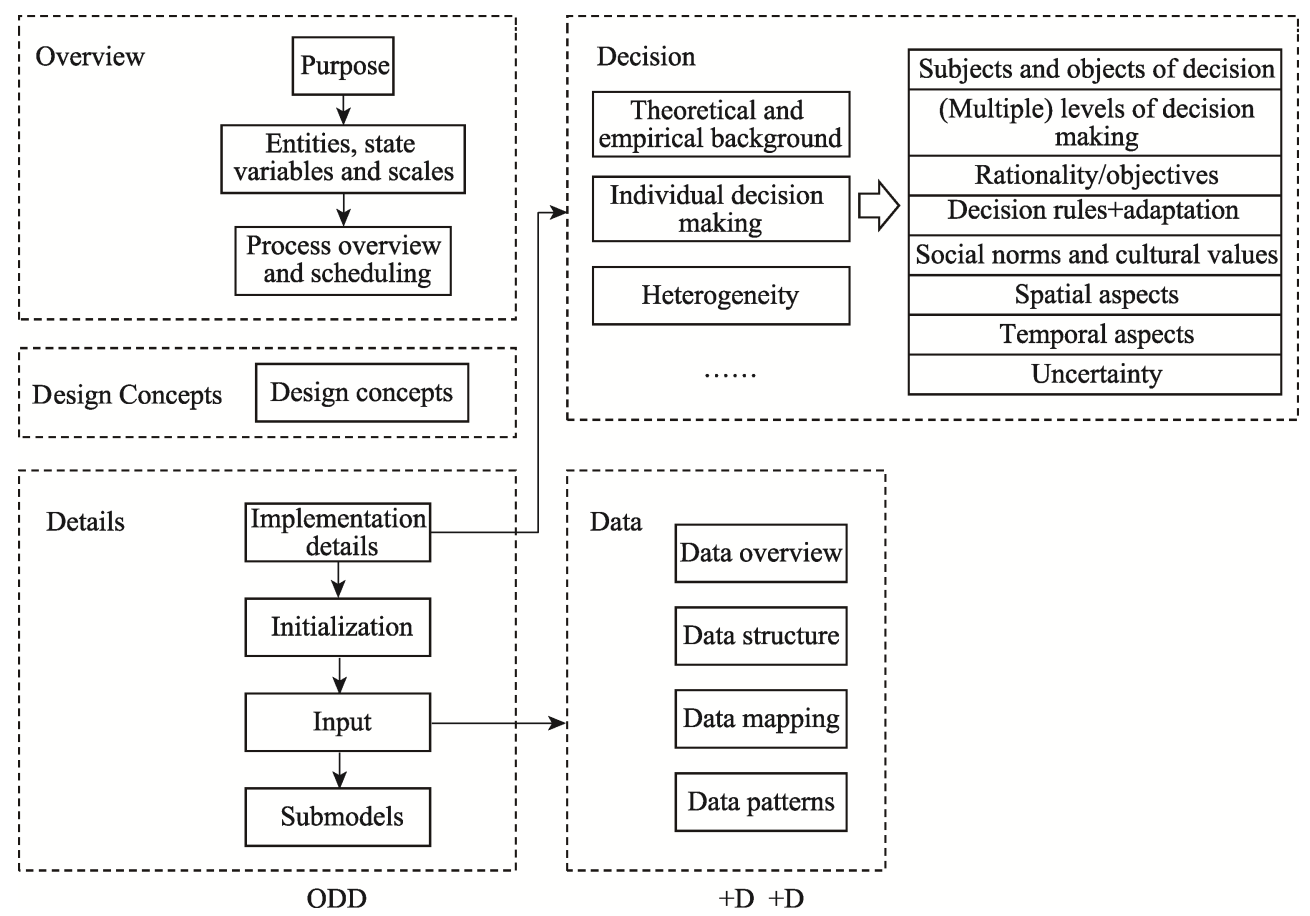

Figure 3 ODD protocol and its extension

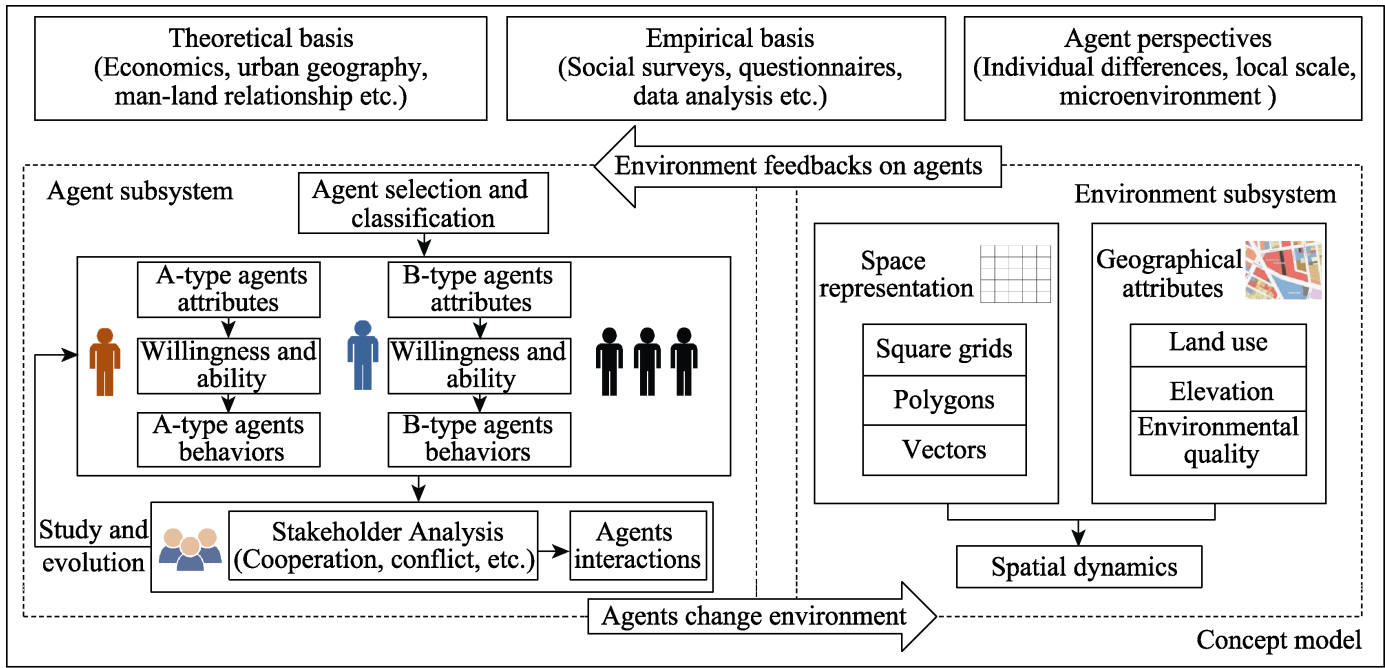

Figure 4 Agent-based modeling framework from the agent perspective

In the agent subsystem, agent selection and classification should be performed first to determine the basic components of the model. Each type of agent is classified according to its attributes. For example, in the urban land change model, residents can be sub-divided based on attributes like income and age. In the agricultural land model, farmers can be classified by full-time or part-time, whether they fall into the category of labor force. Then the deci- 
sion-making willingness and ability of each type of agents are determined according to the attributes. The ability determines the possible decision-making options in a period of time, and the willingness determines the preference for specific options of the agents (Valbuena et $a l ., 2010 \mathrm{c}$ ). The last step of the agent subsystem building is to represent the corresponding decision rules for each type of agents, and the interactions between different agents such as cooperation, competition and learning.

The land system is a spatial system, so building ABMs for land systems requires the design of environment subsystems. First, in order to represent the environment, we can choose CA square girds, polygon grids or vector layers. Then environment units should be given geographical attributes, such as land cover/use type, altitude, environmental quality, etc.

Both agent subsystem and environment subsystem change dynamically. The update of the environment subsystem is driven by natural changes and agents' influence on the environment. The update of agent system is affected by the learning and evolution of agents on one hand, and by the environment's feedback on the other hand. By characterizing agent features, behaviors and interactions and representation of environment a conceptual ABM of land system is built.

Based on the conceptual model, decision rules should be formulated as mathematically as possible to build a theoretical model. Further, a computer model can be constructed with a modeling platform (or independent development using a programming language). Before

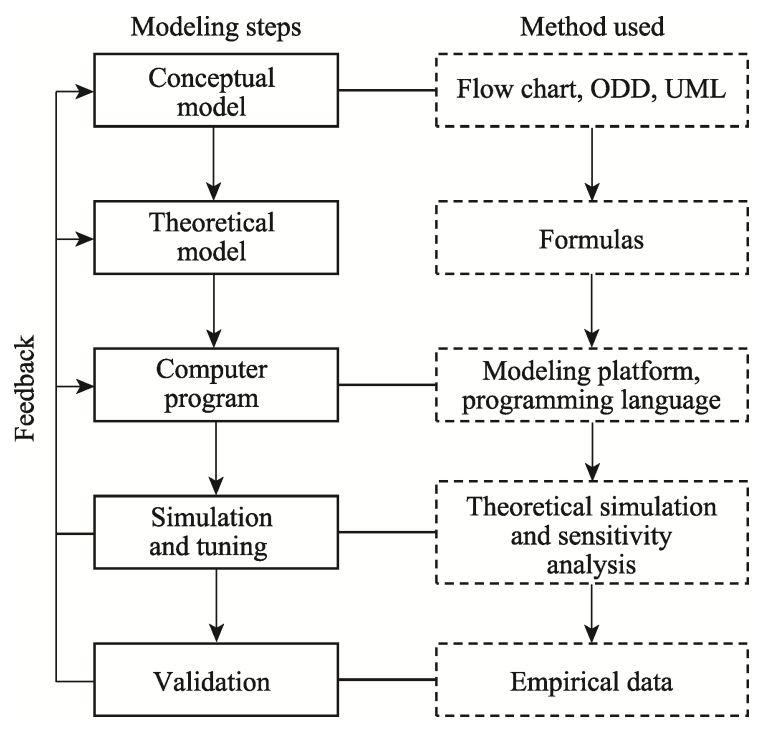

Figure 5 Modeling procedure and related approach of ABM application, the model should be checked by sweeping the parameters. Theoretical simulation can help reveal whether the characteristics shown by the model are consistent with assumptions and common sense. At the same time, sensitivity analysis can be performed. Then the model can be parameterized with empirical data, and the actual land system change can be simulated. The modeling results should be compared with actual data like remote sensing data to validate the model (Figure 5). It should be noted that this modeling procedure is not one-way, and problems in the later steps may need corrections of previous steps.

\section{Discussion}

The ABM simulates land change drivers through representing decision-making behaviors of micro agents. Macro land change and spatial patterns are emergent results. In this way, the $\mathrm{ABM}$ has its irreplaceable role in all these land system modeling approaches. Its value lies not in higher simulation accuracy, but in its ability to promote a better understanding of land change mechanisms and processes. Due to its multi-source nature, the ABM is different in 
model design, programming, parameterization, calibration and implementation from traditional methods. There are also many challenges, such as representation of agents' behaviors, application in large scale, verification and model reuse.

\subsection{Strengthen the representation of agent behaviors}

The core of the agent-based modeling is the design of agent behavior rules. How to better capture the agent behaviors is a key issue for ABM researchers. There are many different ways to characterize behaviors of agents such as the commonly used utility functions and statistical methods. Genetic algorithms and neural networks have also been integrated into ABM to define agent behaviors. When multiple methods are coupled, it is necessary to solve the spatial and temporal inconsistency. Agent classification can aggregate agents into class, further defining the behaviors of each class. In addition, it is necessary to consider the agent's ability to perceive the environment, whether the agent is fully or bounded rational. The integration of sociological and psychological theories helps to characterize the agent's cognitive, emotional, and belief dynamics. Another important thing to consider is to find balance between theoretical basis and empirical observation (Filatova et al., 2013).

In representing the behavior of agents, the detailed description of behaviors in economic models (such as the process of expectation formation and proactive behavior) has not been well integrated into the land change ABM. More work needs to focus on establishing links between spatial economic models and land change ABM. The parameterizing process of agent decision-making simulation urgently requires standardized methods to improve efficiency (NRC, 2014). In addition, most current ABM research focuses on direct agents of land use, such as farmers and urban residents. Recent studies show that large-scale land acquisitions, contract farming, and investment by remote entities cannot be ignored (Verburg et al., 2019).

\subsection{Application of $\mathrm{ABM}$ at larger scales}

Large-scale ecological models and climate models have been widely used. Biophysical processes are well represented, while human activities are often oversimplified in the models. Coupling the ABM with the ecological model is a possible solution. However, most of current $\mathrm{ABMs}$ focus on the local scale. Local decision rules in the model cannot be directly applied to a larger spatial range or a finer spatial resolution (Rounsevell et al., 2012). In addition, the large amount of empirical data based on local survey required for simulation has limited the application of ABM to regional and global scales (Verburg et al., 2019). When applying to regional and larger scales, the ABM becomes more difficult at representation of the diversity of decisions. Some researchers have attempted to develop regional-scale ABMs, but effective solutions are still lacking (Castella and Verburg, 2007; Valbuena et al., 2010c; Rounsevell et al., 2014).

\subsection{Validation of ABM}

Model validation checks the validity and its degree of the model by comparing the simulation results with actual data, which is an important part of the model evaluation. Validation of land change models includes pattern validation and structural validation. The pattern va- 
validation is to compare the simulated land use patterns with the actual land use patterns. The actual land use patterns can be obtained by classification of remote sensing data. Methods like point-to-point comparison, kappa coefficient, and landscape index are commonly used. Structural validation is to justify the rationality of the model mechanism and process, which is still a challenge (van Vliet et al., 2016). The main reason is that although the pattern of land use is observable, the process and mechanism of the pattern formation are difficult to observe and quantitatively represent.

\subsection{Reusability}

From the perspective of the construction process of the ABM, the reusability of $A B M s$ is poor. A specific model cannot usually be applied to other regions. Compared with simple abstract models, highly complex and data-dependent models are more opaque to users (O'Sullivan et al., 2016). A large number of case studies are difficult to generalize. O'Sullivan et al. (2016) call it YAAWN ("Yet another agent-based model... Whatever... Never mind...") syndrome. Different researchers have different understandings of ABM ideas, so they design ABM to solve specific problems that already exist in their brains; and ABMs require a large amount of data at both the level of micro agents' features and the level of aggregated results applied to specific backgrounds. For research problems that can be explained by simple analytical models, and research problems that focus on prediction rather than structural interpretation, researchers should consider whether ABM is needed and the large amount of investment it requires (NRC, 2014).

In summary, the land system $\mathrm{ABM}$ has unique advantages and significant positions, but there are still a lot of challenges, and its methodology is still in the process of formation. This means that land system ABM needs more attention from researchers and modelers; on the other hand, researchers also need to take a cautious attitude when adopting this method.

\section{References}

Allan R J, 2010. Survey of Agent Based Modelling and Simulation Tools. Science \& Technology Facilities Council.

An L, 2012. Modeling human decisions in coupled human and natural systems: Review of agent-based models. Ecological Modelling, 229: 25-36.

An L, Linderman M, Qi J et al., 2005. Exploring complexity in a human-environment system: An agent-based spatial model for multidisciplinary and multiscale integration. Annals of the Association of American Geographers, 95(1): 54-79.

An L, Zvoleff A, Liu J G et al., 2014. Agent-based modeling in coupled human and natural systems (CHANS): Lessons from a comparative analysis. Annals of the Association of American Geographers, 104(4): $723-745$.

Ascensao F, Clevenger A, Santos-Reis M et al., 2013. Wildlife-vehicle collision mitigation: Is partial fencing the answer? An agent-based model approach. Ecological Modelling, 257: 36-43.

Bakker M M, Alam S J, van Dijk J et al., 2015. Land-use change arising from rural land exchange: An agent-based simulation model. Landscape Ecology, 30(2): 273-286.

Batty M, 2001a. Agent-based pedestrian modeling: Editorial. Environment and Planning B-Planning \& Design, 28(3): 321-326.

Batty M, 2001b. Polynucleated urban landscapes. Urban Studies, 38(4): 635-655.

Batty M, Desyllas J, Duxbury E, 2003. The discrete dynamics of small-scale spatial events: Agent-based models of mobility in carnivals and street parades. International Journal of Geographical Information Science, 17(7): 
673-697.

Benenson I, Torrens P M, 2004. Geosimulation: Automata-Based Modeling of Urban Phenomena. John Wiley \& Sons.

Bert F E, Podesta G P, Rovere S L et al., 2011. An agent based model to simulate structural and land use changes in agricultural systems of the argentine pampas. Ecological Modelling, 222(19): 3486-3499.

Bone C, Dragicevic S, 2010. Simulation and validation of a reinforcement learning agent-based model for multi-stakeholder forest management. Computers Environment and Urban Systems, 34(2): 162-174.

Brady M, Kellermann K, Sahrbacher C et al., 2009. Impacts of decoupled agricultural support on farm structure, biodiversity and landscape mosaic: Some EU results. Journal of Agricultural Economics, 60(3): 563-585.

Bravo M, Briceno L, Cominetti R et al., 2010. An integrated behavioral model of the land-use and transport systems with network congestion and location externalities. Transportation Research Part B-Methodological, 44(4): 584-596.

Brown D G, Robinson D T, 2006. Effects of heterogeneity in residential preferences on an agent-based model of urban sprawl. Ecology and Society, 11(1): 22.

Brown D G, Verburg P H, Pontius R G et al., 2013. Opportunities to improve impact, integration, and evaluation of land change models. Current Opinion in Environmental Sustainability, 5(5): 452-457.

Cabral R B, Geronimo R C, Lim M T et al., 2010. Effect of variable fishing strategy on fisheries under changing effort and pressure: An agent-based model application. Ecological Modelling, 221(2): 362-369.

Castella J C, Trung T N, Boissau S, 2005. Participatory simulation of land-use changes in the northern mountains of Vietnam: The combined use of an agent-based model, a role-playing game, and a geographic information system. Ecology and Society, 10(1): 32.

Castella J C, Verburg P H, 2007. Combination of process-oriented and pattern-oriented models of land-use change in a mountain area of Vietnam. Ecological Modelling, 202(3/4): 410-420.

Chen X D, Lupi F, An L et al., 2012. Agent-based modeling of the effects of social norms on enrollment in payments for ecosystem services. Ecological Modelling, 229: 16-24.

Chen X D, Vina A, Shortridge A et al., 2014. Assessing the effectiveness of payments for ecosystem services: An agent-based modeling approach. Ecology and Society, 19(1): 15.

Dahal K R, Chow T E, 2014. An agent-integrated irregular automata model of urban land-use dynamics. International Journal of Geographical Information Science, 28(11): 2281-2303.

Dai E F, Ma L, 2018. Review on land change modeling approaches. Progress in Geography, 37(1): 152-162. (in Chinese)

Deadman P, Robinson D, Moran E et al., 2004. Colonist household decisionmaking and land-use change in the Amazon rainforest: An agent-based simulation. Environment and Planning B-Planning \& Design, 31(5): 693-709.

Dion E, VanSchalkwyk L, Lambin E F, 2011. The landscape epidemiology of foot-and-mouth disease in South Africa: A spatially explicit multi-agent simulation. Ecological Modelling, 222(13): 2059-2072.

Farmer J D, Foley D, 2009. The economy needs agent-based modelling. Nature, 460(7256): 685-686.

Filatova T, Parker D, van der Veen A, 2009a. Agent-based urban land markets: Agent's pricing behavior, land prices and urban land use change. JASSS: The Journal of Artificial Societies and Social Simulation, 12(1): 29.

Filatova T, van der Veen A, Parker D C, 2009b. Land market interactions between heterogeneous agents in a heterogeneous landscape-tracing the macro-scale effects of individual trade-offs between environmental amenities and disamenities. Canadian Journal of Agricultural Economics: Revue Canadienne D Agroeconomie, 57(4): 431-457.

Filatova T, Verburg P H, Parker D C et al., 2013. Spatial agent-based models for socio-ecological systems: Challenges and prospects. Environmental Modelling \& Software, 45: 1-7.

Green D G, Sadedin S, 2005. Interactions matter: Complexity in landscapes and ecosystems. Ecological Complexity, 2(2): 117-130.

Grimm V, 1999. Ten years of individual-based modelling in ecology: What have we learned and what could we learn in the future? Ecological Modelling, 115(2/3): 129-148. 
Grimm V, Berger U, Bastiansen F et al., 2006. A standard protocol for describing individual-based and agent-based models. Ecological Modelling, 198(1/2): 115-126.

Grimm V, Revilla E, Berger U et al., 2005. Pattern-oriented modeling of agent-based complex systems: Lessons from ecology. Science, 310(5750): 987.

Haase D, Haase A, Kabisch $\mathrm{N}$ et al., 2012. Actors and factors in land-use simulation: The challenge of urban shrinkage. Environmental Modelling \& Software, 35: 92-103.

Haase D, Lautenbach S, Seppelt R, 2010. Modeling and simulating residential mobility in a shrinking city using an agent-based approach. Environmental Modelling \& Software, 25(10): 1225-1240.

Huang Q X, Parker D C, Sun S P et al., 2013. Effects of agent heterogeneity in the presence of a land-market: A systematic test in an agent-based laboratory. Computers Environment and Urban Systems, 41: 188-203.

Janssen M A, Alessa L N i, Barton M et al., 2008. Towards a community framework for agent-based modelling. Journal of Artificial Societies and Social Simulation, 11(2): 6.

Johnson P A, Sieber R E, 2011. Negotiating constraints to the adoption of agent-based modeling in tourism planning. Environment and Planning B-Planning \& Design, 38(2): 307-321.

Johnston K M, 2013. Agent-based Modeling in ArcGIS. California: Esri Press.

Kocabas V, Dragicevic S, 2009. Agent-based model validation using Bayesian networks and vector spatial data. Environment and Planning B-Planning \& Design, 36(5): 787-801.

Laatabi A, Marilleau N, Nguyen-Huu T et al., 2018. ODD+ 2D: An ODD based protocol for mapping data to empirical ABMs. Journal of Artificial Societies and Social Simulation, 21(2): 1-9.

Lambin E F, Geist H J, 2006. Land-use and land-cover change: Local processes and global impacts. Springer Science \& Business Media.

Le Page C, Bazile D, Becu N et al., 2013. Agent-based modelling and simulation applied to environmental management. In: Edmonds B, Meyer R (eds.). Simulating Social Complexity: A Handbook. Springer Berlin Heidelberg, Berlin, Heidelberg, 499-540.

Le Q B, Park S J, Vlek P L G et al., 2008. Land-Use Dynamic Simulator (LUDAS): A multi-agent system model for simulating spatio-temporal dynamics of coupled human-landscape system: I. Structure and theoretical specification. Ecological Informatics, 3(2): 135-153.

Li S Y, Li X, Liu X P et al., 2013. Simulation of spatial population dynamics based on labor economics and multi-agent systems: A case study on a rapidly developing manufacturing metropolis. International Journal of Geographical Information Science, 27(12): 2410-2435.

Li S Y, Liu X P, Li X et al., 2017. Simulation model of land use dynamics and application: Progress and prospects. Journal of Remote Sensing, 21(3): 329-340. (in Chinese)

Li X, Liu X P, 2007. Defining agents' behaviors to simulate complex residential development using multicriteria evaluation. Journal of Environmental Management, 85(4): 1063-1075.

Li X, Liu X P, 2008. Embedding sustainable development strategies in agent-based models for use as a planning tool. International Journal of Geographical Information Science, 22(1): 21-45.

Little L R, Punt A E, Mapstone B D et al., 2009. An agent-based model for simulating trading of multi-species fisheries quota. Ecological Modelling, 220(23): 3404-3412.

Liu X P, Li X, Yeh A G O, 2006. Multi-agent systems for simulating spatial decision behaviors and land-use dynamics. Science in China Series D-Earth Sciences, 49(11): 1184-1194.

Müller D, Munroe D K, 2014. Current and future challenges in land-use science. Journal of Land Use Science, 9(2): 133-142.

Macal C M, North M J, 2010. Tutorial on agent-based modelling and simulation. Journal of Simulation, 4(3): 151-162.

Magliocca N, Safirova E, McConnell V et al., 2011. An economic agent-based model of coupled housing and land markets (CHALMS). Computers, Environment and Urban Systems, 35(3): 183-191.

Mao X Y, Meng J J, Wang Q, 2014. Modeling the effects of tourism and land regulation on land-use change in tourist regions: A case study of the Lijiang River Basin in Guilin, China. Land Use Policy, 41: 368-377.

Muller B, Bohn F, Dressler G et al., 2013. Describing human decisions in agent-based models-ODD plus D, an 
extension of the ODD protocol. Environmental Modelling \& Software, 48: 37-48.

Murray-Rust D, Robinson D T, Guillem E et al., 2014. An open framework for agent based modelling of agricultural land use change. Environmental Modelling \& Software, 61: 19-38.

National Research Council (NRC), 2014. Advancing Land Change Modeling: Opportunities and Research Requirements. National Academies Press.

North M J, Macal C M, 2007. Managing Business Complexity: Discovering Strategic Solutions with Agent-based Modeling and Simulation. Oxford: Oxford University Press.

O'Sullivan D, Evans T, Manson S et al., 2016. Strategic directions for agent-based modeling: Avoiding the YAAWN syndrome. Journal of Land Use Science, 11(2): 177-187.

Parker D C, Brown D G, Polhill J G et al., 2008. Illustrating a new conceptual design pattern for agent-based models of land use via five case studies: The MR POTATOHEAD framework. In: Paredes A L, Iglesias C H (eds). Agent-Based Modelling in Natural Resource Management. Universidad de Valladolid, Valladolid, Spain, 23-51.

Parker D C, Filatova T, 2008. A conceptual design for a bilateral agent-based land market with heterogeneous economic agents. Computers Environment and Urban Systems, 32(6): 454-463.

Parker D C, Manson S M, Janssen M A et al., 2003. Multi-agent systems for the simulation of land-use and land-cover change: A review. Annals of the Association of American Geographers, 93(2): 314-337.

Perez L, Dragicevic S, 2010. Modeling mountain pine beetle infestation with an agent-based approach at two spatial scales. Environmental Modelling \& Software, 25(2): 223-236.

Robinson D T, Sun S P, Hutchins M et al., 2013. Effects of land markets and land management on ecosystem function: A framework for modelling exurban land-change. Environmental Modelling \& Software, 45: $129-140$.

Rounsevell M, Arneth A, Brown D et al., 2012. Incorporating human behaviour and decision making processes in land use and climate system models. GLP Report.

Rounsevell M D A, Arneth A, Alexander P et al., 2014. Towards decision-based global land use models for improved understanding of the Earth system. Earth System Dynamics, 5(1): 117-137.

Schluter M, Pahl-Wostl C, 2007. Mechanisms of resilience in common-pool resource management systems: An agent-based model of water use in a river basin. Ecology and Society, 12(2): 23.

Schmit C, Rounsevell M D A, 2006. Are agricultural land use patterns influenced by farmer imitation? Agriculture Ecosystems \& Environment, 115(1-4): 113-127.

Schreinemachers P, Berger T, 2011. An agent-based simulation model of human-environment interactions in agricultural systems. Environmental Modelling \& Software, 26(7): 845-859.

Schulze J, Müller B, Groeneveld J et al., 2017. Agent-based modelling of social-ecological systems: Achievements, challenges, and a way forward. Journal of Artificial Societies and Social Simulation, 20(2).

Shin J K, Fossett M, 2008. Residential segregation by hill-climbing agents on the potential landscape. Advances in Complex Systems, 11(6): 875-899.

Sun S P, Parker D C, Huang Q X et al., 2014. Market impacts on land-use change: An agent-based experiment. Annals of the Association of American Geographers, 104(3): 460-484.

Sun Z L, Muller D, 2013. A framework for modeling payments for ecosystem services with agent-based models, Bayesian belief networks and opinion dynamics models. Environmental Modelling \& Software, 45: 15-28.

Tan R H, Liu Y L, Zhou K H et al., 2015. A game-theory based agent-cellular model for use in urban growth simulation: A case study of the rapidly urbanizing Wuhan area of central China. Computers Environment and Urban Systems, 49: 15-29.

Tian G J, Qiao Z, 2014. Modeling urban expansion policy scenarios using an agent-based approach for Guangzhou Metropolitan Region of China. Ecology and Society, 19(3): 14.

Topping C J, Hoye T T, Olesen C R, 2010. Opening the black box: Development, testing and documentation of a mechanistically rich agent-based model. Ecological Modelling, 221(2): 245-255.

Turner B L, II, Lambin E F, Reenberg A, 2007. The emergence of land change science for global environmental change and sustainability. Proceedings of the National Academy of Sciences of the United States of America, 
104(52): 20666-20671.

Valbuena D, Bregt A K, McAlpine C et al., 2010a. An agent-based approach to explore the effect of voluntary mechanisms on land use change: A case in rural Queensland, Australia. Journal of Environmental Management, 91(12): 2615-2625.

Valbuena D, Verburg P, Veldkamp A et al., 2010b. Effects of farmers' decisions on the landscape structure of a Dutch rural region: An agent-based approach. Landscape and Urban Planning, 97(2): 98-110.

Valbuena D, Verburg P H, Bregt A K et al., 2010c. An agent-based approach to model land-use change at a regional scale. Landscape Ecology, 25(2): 185-199.

van Vliet J, Bregt A K, Brown D G et al., 2016. A review of current calibration and validation practices in land-change modeling. Environmental Modelling \& Software, 82: 174-182.

Verburg P H, Alexander P, Evans T et al., 2019. Beyond land cover change: Towards a new generation of land use models. Current Opinion in Environmental Sustainability, 38: 77-85.

Verburg P H, Crossman N, Ellis E C et al., 2015. Land system science and sustainable development of the earth system: A global land project perspective. Anthropocene, 12: 29-41.

Verburg P H, Erb K-H, Mertz O et al., 2013. Land system science: Between global challenges and local realities. Current Opinion in Environmental Sustainability, 5(5): 433-437.

Verburg P H, Schot P P, Dijst M J et al., 2004. Land use change modelling: Current practice and research priorities. GeoJournal, 61(4): 309-324.

Villamor G B, Noordwijk M, 2016. Gender specific land-use decisions and implications for ecosystem services in semi-matrilineal Sumatra. Global Environmental Change-Human and Policy Dimensions, 39: 69-80.

Wahyudi A, Liu Y, Corcoran J, 2019. Generating different urban land configurations based on heterogeneous decisions of private land developers: An agent-based approach in a developing country context. ISPRS International Journal of Geo-Information, 8(5): 229.

Wolfram S, 2002. A New Kind of Science. Wolfram Media Champaign.

Yang W S, 2019. Construction and application of an agent-based regional land use change model (APUS): A case study in Tongdu Town, Kunming [D]. Guangzhou: Sun Yat-sen University. (in Chinese)

Yu Q Y, Wu W B, Tang H J et al., 2011. Complex system theory and agent-based modeling: Progresses in land change science. Acta Geographica Sinica, 66(11): 1518-1530. (in Chinese)

Zhang H H, Zeng Y N, Bian L, 2010. Simulating multi-objective spatial optimization allocation of land use based on the integration of multi-agent system and genetic algorithm. International Journal of Environmental Research, 4(4): 765-776.

Zhou C H, Ou Y, Ma T et al., 2009. Theoretical perspectives of CA-based geographical system modeling. Progress in Geography, 28(6): 833-838. (in Chinese) 